\title{
Rire comme une folle... Sur d'éventuels effets politiques de la parodie La Tour de la Défense de Copi
}

\section{Olivier Neveux}

\section{Q OpenEdition}

\section{Journals}

Édition électronique

URL : http://journals.openedition.org/recherchestravaux/304

DOI : 10.4000/recherchestravaux.304

ISSN : 1969-6434

Éditeur

UGA Éditions/Université Grenoble Alpes

\section{Édition imprimée}

Date de publication : 15 octobre 2006

Pagination : 77-89

ISBN : 978-2-84310-099-4

ISSN : 0151-1874

Référence électronique

Olivier Neveux, «Rire comme une folle... Sur d'éventuels effets politiques de la parodie La Tour de la Défense de Copi », Recherches \& Travaux [En ligne], 69 | 2006, mis en ligne le 18 décembre 2013,

consulté le 08 septembre 2020. URL : http://journals.openedition.org/recherchestravaux/304 ; DOI : https://doi.org/10.4000/recherchestravaux.304 


\section{Rire comme une folle... Sur d'éventuels effets politiques de la parodie La Tour de la Défense de Copi}

LUC: Est-ce que tu vas t'arrêter? Parler de sperme, franchement... Tu te crois dans un salon mondain, enfin?

COPI $^{\mathrm{I}}$

Je n'ai pas écrit sur ce processus de dénaturalisation pour le simple plaisir de jouer avec la langue ou pour nous obliger à jouer aux marionnettes au lieu d'affronter la «vraie» politique, comme certain-e-s l'ont supputé (comme si le théâtre et la politique étaient toujours des domaines distincts).

J. BUTLER ${ }^{2}$

À la suite, entre autres, de Monique Wittig³, il est possible de désigner l'hétérosexualité comme une injonction, fondamentalement idéologique, c'est-à-dire incorporée, contraignante, structurante et, par définition, invisibilisée et naturalisée. De ceci se déduit, entre autres, un régime spécifique d'organisation de la sensibilité. Nous sommes saisi-e-s par les codes de régulation de l'hétéronormativité (qu'il faudrait en outre et bien évidemment articuler à des considérations classistes, sexistes et «racialistes»). S'il a existé des dramaturgies réfractaires à l' "idéologie hétérosexuelle», ouvertement émancipatrices, souvent explicites et frontales, d'autres ont tenté d'aborder, de biais, les mécanismes de l'assujettissement. Tel est certainement l'un des apports de l'œuvre de Copi : ouvrir la possibilité d'un théâtre qui emprunterait ses codes à l'idéologie dominante et l'entraînerait en un endroit que celui-ci ne peut contenir. Ou :

I. Copi, «La Tour de la Défense» dans Copi, Les Quatre Jumelles / La Tour de la Défense, Paris, Christian Bourgois, I999, p. 58. La pièce sera désormais référencée sous l'abréviation TD.

2. J. Butler, Trouble dans le genre. Pour un féminisme de la subversion (1990), préface de E. Fassin, trad. de l'anglais par C. Kraus, Paris, La Découverte, 2005, p. 43.

3. M. Wittig, La Pensée straight, Paris, Balland, «Le Rayon/Modernes», $200 \mathrm{I}$. 
permettre à une résistance de "glisser», tant bien que mal, dans l'opacité de la domination, "d'un ordre à un autre, en suivant les failles ${ }^{4}$ ".

Une telle lecture de Copi prend acte des singularités et de l'historicité des luttes homosexuelles tout autant que des acquis pratiques et théoriques militants 5 . Chaque fois les œuvres émancipatrices sont tributaires des mouvements auxquels elles s'adossent, qu'elles accompagnent ou, plus rarement, qu'elles devancent. Un tel projet croit percevoir dans tout un pan occulté des productions théâtrales des moyens de contrarier voire de renverser la donne, d'y découvrir pour celles et pour ceux que ce présent effraie et opprime des armes, des tactiques pour en fissurer la massivité. Si l'œuvre de Copi s'extrait du militantisme - de ce que la doxa a fait du terme -, son œuvre n'en propose pas moins - comme une incarnation singulière de motifs oxygénants - un cadre propice à de possibles interventions (pour s'y conformer ou s'en inspirer).

À l'opposé des dramaturgies de la «victimisation» ou des processus tactiques de conformation sociale lorsque le gay et la lesbienne sont invité-e-s à se rendre transparent-e-s, à s'intégrer au dispositif hétérosexuel jusqu'à en épouser les modes de régulation, Copi s'arrache à l'opposition héritée des typologies psychiatriques: hétérosexuel/homosexuel, et à la production positive, constructive, d'une «image de soi » gay, socialement valorisable. Il tend à promouvoir la figure absente de l'homosexualité pacifiée, "présentable»: la folle. Une folle réfractaire aux représentations homophobes de La Cage aux folles $^{6}$ : visions hétérocentrées qui ne font que redoubler les stigmatisations, les marqueurs de discrimination, répondre à la demande hétérosexuelle. Parmi tant d'autres (in)définitions, "être folle» sera ici entendue, à partir de la belle proposition, politique, de l'une d'entre elles, Michel Cressole, au cour de l'hécatombe des années $1980^{7}$ :

[...] c'est appartenir à une sorte de civilisation en exil, comme une immigrée de la sexualité dominante, aussi complètement frustrée qu'un Zoulou, un jeune Palestinien, ou un GI Noir dans le désert d'Arabie Saoudite, qui sont les jeunes hommes avec qui elle aimerait vivre.

4. G. Hocquenghem, L’Après-mai des faunes, préface de G. Deleuze, Paris, Grasset, I974, p. 193.

5. Voir, par exemple, X. Crettiez, sous la dir. de I. Sommier, La France rebelle, Paris, Michalon, 2002, p. 44I-456.

6. J. Poiret, La Cage aux folles, L'Avant-scène, nº $518,1973$.

7. M. Cressole, Une folle à sa fenêtre. Chroniques. L’Autre journal I9go-I99I (1992), GayKitsch-Camp, "Questions de genre», 1996, quatrième de couverture. Les militant-e-s du Front homosexuel d'action révolutionnaire (FHAR) écrivaient en 1971: «Folles: nos frères. Les homo-flics comme les hétéros-flics leur reprochent d'être efféminés, maniérés, de s'afficher. Objet de mépris pour beaucoup de gens, les folles ne sont acceptées que si elles s'amusent (notamment dans les milieux des arts et des lettres)»(FHAR, Rapport contre la normalité, Champ libre, 197I, p. I4). 
Joyeuse ou meurtrie, la folle se définit ainsi tout à la fois dans l'exil spatial et historique - hors du continent hétérosexuel - et dans le regroupement diasporique, dans l'avènement d'une grégarité protéiforme de dissidences. La folle est inadaptée à l'Ordre - symbolique ou moral -, elle tranche avec l'ordinaire d'un monde qu' elle insupporte à ne pouvoir/vouloir s'y conformer. Discrète ou ostentatoire, elle est toujours trop visible, inaccordée aux jeux des représentations. Car la folle est un être désirant - et ce désir (ne pas refréner telle attitude ou telle expérience, espérance érotique) est précisément ce qui l'exclut.

\section{Les «tapettes sur l'estrade ${ }^{8}$ »}

La pièce de Copi, La Tour de la Défense ${ }^{9}$, use des signes du théâtre dit de boulevard. Il faut en préalable, pour percevoir le geste entrepris par Copi, replacer ce théâtre dans l'économie générale de fabrication de la sensibilité - les mythes sur lesquelles perdure la bourgeoisie et notamment l' «idéologie hétérosexuelle». Le boulevard en tant que forme de théâtre "populaire» participe d'une avalisation des dispositifs de régulation du désir, de hiérarchisation des comportements sexuels, de formalisation des attitudes et des comportements. Sans exagérer ses pouvoirs aujourd'hui, sans dédouaner par ailleurs d'autres formes théâtrales, plus aristocratiques, mais tout aussi réactionnaires, le théâtre dit de boulevard sera envisagé simultanément comme agent et symptôme, comme fait idéologique (soit comme "conscience déformée des réalités, et en tant que tels des facteurs réels exerçant en retour une réelle action déformante $\left.{ }^{\mathrm{I0}} »\right)$.

Le boulevard, pour être hétérogène dans ses développements, n'en revient pas moins, chaque fois, à une inspiration initiale:

Qu'on traite des artistes, des députés, des affairistes ou des ouvriers en révolte, on ne peut s'empêcher d'articuler le sujet avec une histoire d'amour (très liée évidemment aux mœurs du temps) et, conjointement et paradoxalement, de chercher à élever le débat en dégageant l'universel du particulier, la leçon générale de l'aventure contingente. ${ }^{\text {II }}$

Ce "genre» théâtral essentialise immanquablement un type de relation, hétérosexuelle, et normativise à cette aune les conduites amoureuses et érotiques $^{\mathrm{I} 2}$. Au-delà de son seul «dire», explicite, de sa morale, la conduite de son

8. J'emprunte l'expression à un sous-titre de G. Chauncey, Gay New York. I890-I940 [1994], Paris, Fayard, 2003, p. 375 .

9. La pièce est publiée en 1978 chez Christian Bourgois et créée par C. Confortès en I98I.

IO. G. Debord, La Société du spectacle [1967], Paris, Gallimard, "Folio», I992, p. 203.

II. M. Corvin, Le Théâtre de boulevard, Paris, Presses universitaires de France, "Que sais-je?", I989, p. Iо.

I2. Voir sur la prédominance du modèle bourgeois, B. Brunet, «Un reflet du monde bourgeois ", Le Théatre de boulevard, Paris, Armand Colin, 2005, p. 16-20. 
intrigue nécessite la validation de ses présupposés, l'admission de ses postulats: la valeur du mariage, le sexisme des figures, etc. L'organisation de la narration, si elle n'épouse pas nécessairement les données aristotéliciennes du possible et du vraisemblable, est surdéterminée par la norme sociale ${ }^{\mathrm{r}}$. Le quiproquo, aussi injuste puisse-t-il paraître, viendra sanctionner un mensonge, une défaillance à la morale majoritaire. Le boulevard fonctionne sur une unité politiquement unificatrice: le cadre dans lequel il s'amorce déroule logiquement, naturellement (i.e. respectueux de la morale dominante) son jeu de conséquences. Unité car il s'agit d'une "pièce bien faite", donnée comme un tout - comme une illusion de totalité. Unificatrice car c'est son mécanisme intrinsèque qui ordonne le jeu des conséquences (aussi invraisemblables soient-elles). Le désordre et les déviations qui semblent prévaloir sont soutenus par une organisation narrative causale et naturalisante. Le montage, aussi invisible soit-il, est idéologique.

Il serait fastidieux d'énumérer tout ce qui, dans La Tour de la Défense, croise le théâtre de boulevard: la réunion en un endroit, un salon bourgeois des années 1970, en une occasion précise, un réveillon, de personnages-prototypes jetés dans une succession d'intrigues parallèles et convergentes, simultanées. L'unité de lieu, de temps, les «bons mots", le rythme effréné, l'enchaînement narratif de péripéties, tout concourt à faire de cette dramaturgie une "pièce bien faite» ouverte aux numéros d'acteurs «hystérisés »... Un soir de réveillon, dans un appartement du quartier de la Défense, un couple en crise reçoit la visite impromptue d'une voisine accompagnée d'un jeune homme et d'une amie. La situation est classique...

Le théâtre de boulevard a ceci de singulier:

Les personnages ne se montrent pas à nous: on nous montre qu'ils se montrent. Ils sont constamment tenus à bout de fil, ces pantins, par une main invisible ellemême, mais tellement facile à deviner dans l'ombre du texte, celle de l'auteur. Le boulevard est un théâtre de montreur. ${ }^{\text {I4 }}$

Derrière chaque personnage: un dramaturge dont la présence sensible se laisse percevoir en toute occasion. La construction de situations prime la

I3. On peut ainsi lire dans le commentaire que Denis Guénoun fait de Yasmina Reza qui ne s'apparente certes pas au boulevard: «Les deux personnages, homme et femme, sont donc mus par deux pulsions bien hétérogènes, puisque l'homme n'est animé que d'un élan qui le ramène à lui, une sorte de poussée qui revient en boucle vers son origine, ce que Deleuze lisant Nietzsche appelle le "retournement sur soi" d'une force initialement lancée vers le dehors, de cette puissance d'extériorisation pure qu'est la vie. Alors que la femme est toute entière occupée de l'autre, tournée ou orientée vers lui, hétérotrope, par cette adresse pleine, sans réserve, à laquelle ne manque que le son de sa propre voix. Ce qui s'avance là sans crier gare est bien une théorie de la différence des sexes, ou plutôt de la différence des genres - car ce n'est pas seulement de sexualité, mais bien de généricité qu'il s'agit, même si la sexualité y est pleinement impliquée.» (D. Guénoun, Avez-vous lu Reza?, Paris, Albin Michel, 2005, p. 98.)

I4. M. Corvin, Le Théâtre de boulevard, op. cit., I24. 
psychologisation et l'autonomie des figures. La surprésence d'un scripteur agençant les faits affirme la prévalence d'un point de vue organisateur, d'une lecture «signée» du réel. Appelons ce scripteur, ici: la folle. La folle décale la situation : le couple (Jean et Luc) est un couple homosexuel; la voisine (Daphnée) est sous acide, mère infanticide; le jeune homme (Ahmed) est arabe, objet des convoitises sexuelles des personnages; l'amie (Micheline) est une "connasse de travelo» (TD, I50). Jean, après avoir "déchir[é]l'anus» (TD, 98) de Luc, lui annonce qu'il le quitte. Daphnée a tué sa fille parce qu'elle la dégoûtait (TD, I7I) : elle l'a entreposée dans une valise pour aller l'enterrer dans le Maine. Un boa surgit des toilettes: il sera dégusté lors du réveillon, tandis qu'une mouette est gavée au caviar et qu'un hélicoptère s'encastre dans une tour. Après avoir souhaité "violer» Micheline dans la baignoire, ce à quoi elle rechigne, Ahmed se révèle in fine être son voisin. Ils restent tous deux, seuls, tandis que les autres accompagnent Daphnée au commissariat. La voiture s'explose dans la tour de la Défense: «Parfois Dieu arrive si soudainement» (TD, I83).

De prime abord, le boulevard de Copi s'apparente à un boulevard "déjanté», progressiste, fort d'une plus-value libérale, provocatrice et subversive: une adaptation culturalisée du boulevard. Les personnages, s'ils sont issus de la bourgeoisie, appartiennent à sa périphérie: couple homosexuel aisé, on y prend de l'acide, fume des pétards, baise, etc. Un boulevard a priori gay, donc, à l'humour communautaire et dissident: un humour camp $p^{15}$.

\section{Le rire camp}

Caractériser un rire, un humour, c'est inévitablement encourir la désapprobation des dominants qui ne rêvent que d'homogénéiser le rire, le pacifier ou le neutraliser et pour cela, le drapent dans une très suspecte universalité, partageable par tou-te-s, communiant dans les mêmes éclats. Susan Sontag

I5. «[Le camp] évoque pêle-mêle l'humour «folle», le travestissement provocant, l'artifice revendiqué, l'autodérision outrageuse, la théâtralisation parodique... Comme le dandysme, dont il est un peu l'avatar postmoderne, le camp est tout autant une esthétique qu'une éthique, une microculture hyper-référencée qu'une stratégie oblique de résistance aux normes. Apparu dans l'argot de l'Angleterre victorienne du début du $x^{e}{ }^{e}$ siècle, peu de temps après le procès d'Oscar Wilde (1895), le mot camp [...] désigne alors la gestuelle exagérée des folles - c'est par le corps et ses postures que l'homosexuel est identifié, stigmatisé. [...] Comme le suggérait déjà Susan Sontag, le camp découvre une forme de relation nouvelle, et plus complexe, avec le sérieux: on peut se moquer du sérieux et prendre la frivolité au sérieux. Ainsi, plus qu'un style et moins qu'une politique, le camp [...] se présente plutôt comme une volonté subversive de mettre en échec toutes les assignations, dans l'assomption d'un éclat de rire.» (P. LebrunCordier, "Camp", dans D. Eribon (sous la dir. de, avec A. Lerch. Coord.: F. Haboury), Dictionnaire des cultures gays et lesbiennes, Paris, Larousse, 2003. Voir, par ailleurs, P. Mauriès, Second Manifeste camp, Paris, Seuil, 1979.) 
affirme, dans ses "Notes on camp» (1964), que "[1]e camp est ésotérique - c'est de l'ordre d'un code intime, peut-être d'une affirmation d'identité adressée à des petites coteries urbaines ${ }^{16}{ }$. Le camp fait ici mémoire de ce que le rire est clivant, localisé, situé; que le rire est soumis à des histoires, des contextes particuliers; qu'il est produit socialement; qu'il comporte peut-être une dimension impartageable.

Le camp, souligne Sontag, n'est pas un procédé mais une sensibilité, une perception du monde, minoritaire, parodique, insaisissable, rétive à toute taxinomie. Tout au plus peut-on en percevoir empiriquement quelques traits distinctifs: une esthétisation du monde, rebelle aux catégories du "beau », du «laid»; une emphase théâtrale dans l'appréhension du réel: «la voie du camp ne s'appréhende pas en termes de beauté mais en termes de degré d'artifice, de stylisation ${ }^{17}$ ». Dix ans plus tard, Patrick Mauriès, dans un Second Manifeste camp, relève quelques traits propres au camp, cet «objet de discours impossible $^{18}{ }$ : un esthétisme qui se connaît et fonctionne par surenchère de légèreté et de superficialité. Le camp se dégage de l'injonction à l'authenticité. Pur artifice, construit, mobile, inflationniste, le camp n'a pas vocation à se pétrifier dans l'art, séparé de l'existence. Il prend d'assaut l'autonomie esthétique pour y imposer la vie. Dès lors, tout devient théâtre - pas moins théâtral, mis en scène que la quotidienneté.

Copi écrit donc un boulevard $c a m p^{19}$ : on s'insulte beaucoup dans La Tour de la Défense, on surenchérit dans la parole "outrageante», les remarques définitives («Mais elle est folle [...] et elle est passive, elle se fait enculer, c'est les plus méchantes!» [TD, IO3]), on y donne des conseils de beauté ("Qu'est-ce que ça la remonte, de se faire baiser! Tu as la peau fraîche et tendue!» [TD, IOO]), on y exhibe ses bijoux, on théâtralise ses déclarations (de rupture ou d'amour)... Rien à voir ici avec le lent pourrissement du rire basculant dans la dérision la plus sinistre ${ }^{20}$ pas plus qu'avec le rire d'accommodement au monde,

I6. Je traduis. Voir S. Sontag, "Notes on camp", Against interpretation and other essays, London, Eyre \& Spottiswoode, 1967, p. 275. " camp is esoteric — something of a private code, a badge of identity even, among small urban cliques."

17. Je traduis. Id., p. 277: "[...] the way of camp, is not in terms of beauty, but in terms of the degree of artifice, of stylisation".

I8. P. Mauriès, Second Manifeste camp, Paris, Seuil, «Fiction \& Cie», I979, p. 9.

19. Cette rencontre entre camp et boulevard est peu surprenante: «Rien n'est plus camp que le théâtre de l'amour, presque son pathos (fût-il très sobre): ne jamais oublier que la sentimentalité est un des grands ressorts du camp.» (P. Mauriès, op. cit., p. 32) et le camp n'a eu de cesse de "puiser» dans la "culture de grande masse» (ibid., p. 2I).

20. «En fait, ce qui au fond fait le plus rire dans ce comique des temps démocratiques, c'est de montrer qu'en toutes occasions tout peut être dit, tout peut être entendu sans que jamais la moindre suite n'y soit donnée. Ce qui fait rire, c'est l'indignation tombée dans l'indifférence, c'est la protestation écoutée avec compassion et vouée à l'impuissance, c'est la démonstration 
le rire camp est dérèglement de la coercitive cohérence politique, sociale et culturelle qui régit le quotidien. Comme le note justement Marie-Hélène Bourcier, le camp s'avère:

[...] une stratégie de résistance culturelle, qui se fonde sur la conscience partagée du fait que l'on est pris dans un système complexe de significations sociales et sexuelles. «Le camp» propose d'y résister de l'intérieur via la parodie, l'exagération, la théâtralisation, la prise au sens littéral des codes tacites qui régissent notre manière de vivre et la représentation (les codes de la masculinité par exemple). ${ }^{21}$

Le camp n'adopte pas une attitude frontale, exogène: il s'inscrit de l'intérieur dans une critique politique des représentations dominantes. La critique a ceci de particulier qu'elle ne s'exonère pas du monde: elle ne choisit pas une hypothétique rive pour, de l'extérieur, asséner sa justesse. Elle contamine, entriste, de l'intérieur le champ des pouvoirs. Pour reprendre l'une des définitions inaugurales de la parodie: le camp chante faux ${ }^{22}$ - et à tue-tête si bon lui semble - et produit alors ou rires empathiques ou consternations et désapprobations... Il se love au cœur de l'idéologie qu'il soumet à l'outrance et à la surenchère, exacerbée dans ses conséquences et son inanité, ses logiques et sa brutalité. In fine,

[c]e qui impose le rejet du camp tient à son fondement même: à la mimétique - à la façon dont ostensiblement, et de manière obscène, il s'offre comme une singerie sans âme, sans vérité, sans identité. C'est cela, si l'on y réfléchit bien, que l'on ne peut admettre - cela, outre la jouissance qui s'éprouve à le montrer - et que l'on censure avec dégoût. ${ }^{23}$

\section{Une parodie de parodie}

Dans son ouvrage Trouble dans le genre, Judith Butler avalise la puissance parodique comme possibilité subversive. Dissociant «le sexe anatomique, l'identité de genre et la performance du genre », l'auteure travaille sur la portée politique des Drag Queen/King: "En imitant le genre, le drag révèle implicitement la structure imitative du genre lui-même - ainsi que sa contingence ${ }^{24}$.» La parodie du genre (féminin ou masculin) que propose le/la drag vient révéler l'absence d'original de tout genre:

de l'horreur sans le moindre espoir qu'il y soit rien changé.» (J.-P. Curnier, Manifeste, Léo Scheer, 2000, p. II.)

2I. M.-H. Bourcier, Queer zones. Politiques des identités sexuelles, des représentations et des savoirs, Paris, Balland, 200I, p. I86.

22. Voir G. Genette, Palimpsestes. La littérature au second degré, Paris, Seuil, «Poétique», 1982, p. I7.

23. P. Mauriès, Second Manifeste camp, op. cit. , p. 56.

24. J. Butler, Trouble dans le genre, op. cit., p. 26I. 
$\mathrm{Au}$ fond, la parodie porte sur l'idée même d'original; tout comme la notion psychanalytique d'identification de genre renvoie au fantasme d'un fantasme [...], la parodie du genre révèle que l'identité originale à partir de laquelle le genre se construit est une imitation sans original. Plus précisément, on a affaire à une production dont l'un des effets consiste à se faire passer pour une imitation. ${ }^{25}$

Le mirage d'une originalité du genre - originalité qui fonde le binarisme et l'assignation de genre - peut être mis en crise grâce à la parodie du genre ${ }^{26}$.

Lire la proposition de Copi à l'aune de l'apport butlerien permet peutêtre de préciser sa radicalité conjoncturelle ${ }^{27}$. Il faut pour cela, au préalable, forcer et outrepasser la proposition de Butler: celle-ci envisage la parodie dans le cadre de l'articulation sexe/genre. Plus largement, ne peut-on percevoir dans la parodie, une "certaine" parodie, un mode d'intervention politique singulier? Et, si le binarisme des genres relève de l'«idéologie hétérosexuelle», ne peut-on retrouver dans ce qui parodierait l'un de ses agents/symptômes idéologiques une semblable défaillance de l'institué? Au commencement: le théâtre de boulevard et ce que celui-ci charrie de lieux communs normatifs et prescriptifs. Copi parodie ce modèle en exhibant le geste même de l'imitation qu'il rend outrancière. Il décode ou surcode le code, à la manière d'un-e drag se construisant à partir des codes de la masculinité ou de la féminité tels qu'ils sont créés, avalisés et contraints dans nos sociétés. Plutôt que de produire un «boulevard gay» qui entérine, de fait, des modes de régulation politico-sexuels, plutôt que d'universaliser ou d'identitariser une forme ce qui, en l'occurrence, reviendrait au même, Copi en trouble jusqu'au vertige la netteté. Il ne s'agit pas de plier des personnages "gays», minoritaires, à la structure du boulevard, de procéder dramaturgiquement à leur assimilation - mais bien d'outrer les enjeux du boulevard, de citer une citation sans modèle (autre que social). La parodie n'est pas caricature condescendante: elle ne cible pas le boulevard en

25. Id., p. 26I.

26. "[...] tout genre est performance. La drag queen de Butler, les performances de genre de Madonna ne sont que la monstration hyperbolique du caractère construit de la féminité - et on pourrait en dire autant de la masculinité. En définissant l'identité sexuelle et le genre comme performatifs, c'est-à-dire comme résultant d'un effet de répétition des codes de performance de genre, Butler dit clairement que l'hétérosexualité, comme tout genre est une parodie, c'est-à-dire une imitation sans original du genre masculin ou féminin». (M. H. Bourcier, Sexpolitiques. Queer Zones 2, Paris, La Fabrique, 2005, p. I36.)

27. Une telle transposition est néanmoins problématique: je ne joue pas ici d'une possible confusion entre les genres (féminin et masculin) et le genre théâtral que serait le boulevard. Ce que Copi parodie, à travers l'ordonnancement spécifique de la sensibilité qu'est le boulevard, est bien plus radical qu'une simple subversion esthétique. Il faudrait par ailleurs affiner à partir de la distinction qu’opère F. Jameson entre «pastiche» et "parodie» («le pastiche, c’est la parodie absolue, la parodie qui a perdu son humour») la position qu'adopte Copi (Voir J. Butler, Trouble dans le genre, op. cit., p. 262). 
tant que $\operatorname{te}^{28}$ mais la somme d'artifices qui le justifient et le conditionnent politiquement. Cette «singerie sans âme, sans vérité, sans identité» peut alors rejaillir sur les prétendues âme, vérité, et identité de ce qui est singé.

De prime abord, Copi déplace les données initiales, charge significativement l' «original» de ce que la société hétéronormative et hygiéniste forclot: sexes et drogues. Ce déplacement est en quelque sorte matérialisé dans la dramaturgie même. Tandis que dans le boulevard "[1]'imagination du spectateur sait où on l'entraîne: au lit, mais ses yeux et ses oreilles restent chastes ou presque, le sens "propre" des mots étant là pour protéger son hypocrisie ${ }^{29}$ ", La Tour de la Défense, elle, entraîne inexorablement l'ouïe du spectateur vers la salle de bain, lieu de la consommation sexuelle, en direct ${ }^{30}$. Le lit, la chambre ne sont plus les repères ultimes de la production érotique... À propos des pratiques Sado/Masochistes, Bourcier note:

Le fait que ces pratiques bizarres aient lieu dans des "situations très inhabituelles", en public et à plusieurs, dans des lieux différents de la chambre à coucher va à l'encontre de l'habituel confinement de la sexualité dans la sphère privée et domestique. [...] les délimitations spatiales et temporelles, les divisions entre public et privé, domestique et institutionnel imposées aux pratiques sexuelles et aux lieux de savoir sur le sexe relèvent d'une architecture politique générale qui inclut la gestion de l'espace et des corps. ${ }^{3 \mathrm{I}}$

Le déplacement dramaturgique opéré par Copi est moins anecdotique qu'il n'y paraît - certes encore bien timide au regard du panel des pratiques sexuelles, mais bien téméraire lorsqu'on le compare à l'immense majorité des propositions théâtrales. Il visibilise le trajet opéré entre le théâtre de boulevard et sa parodie: le sexe n'y est plus caché, pathogène (sauf lorsqu'il vient à manquer), relégué dans un lieu confiné et traditionnel, enjeu de l'intrigue ${ }^{32}$. Le sexe s'inscrit dans une nouvelle territorialisation de l'espace domestique, attenant à l'espace de représentation, il est commenté en direct ou a posteriori. Le sexe n'a plus ni âme ni vérité: il n’est plus au-delà, hors scène et surprésent, mais en proche

28. "Écoutez Copi parler théâtre; il affecte de tenir la technique, le calcul des entrées et des sorties de scène, le typage des personnages, la rapidité du dialogue, pour l'essentiel. Et il les réussit parfaitement, mécaniques merveilleuses, précises et efficaces (est-ce pour rien qu’il admire le boulevard?) » (G. Hocquenghem, "Copi soit-il», dans Copi, Une visite inopportune (I988), Paris, Christian Bourgois, 2003, p. 82.)

29. M. Corvin, Le Théatre de boulevard, op. cit., p. I3.

30. Dans sa très stimulante mise en scène de La Tour de la Défense en 2005, M. Di Fonzo Bo a inclus la salle de bain (et les ébats) dans le champ visuel des spectateurs.

3I. M.-H. Bourcier, Queer zones. Politiques des identités sexuelles, des représentations et des savoirs, op. cit., p. 85-86.

32. Micheline dit à Luc à propos d'Ahmed: "Je l'ai invité chez moi pour ce soir, mais si tu veux tu peux venir le baiser demain à l'heure du thé, j'en aurai déjà marre, je te le donnerai. Tu as vu comme il est monté?» (TD, I07) 
périphérie, contingent, dédramatisé. Un déplacement «sensible», donc, mais qui ne mène "nulle part» (si ce n'est, au final, littéralement, dans un mur...) Il est en effet impossible de resémantiser politiquement ce qui se joue - à la notable exception de ses éventuels effets, en situation. Saturée de signes boulevardiers, la pièce est construite sur une accumulation qui a paradoxalement valeur soustractive: elle révèle, par ricochet, organiquement lié à lui, la vacance «ontologique» et le trop plein social qui soutient le boulevard.

La dramaturgie de Copi rend ainsi évidente la précarité crispée des systèmes d'agencement causaux qui régissent la vie sociale. Précaire et arbitraire: Copi "dénature» l'amour, la conjugalité, ou, plus butlerien encore, le genre (le travestissement de Micheline ne fait pas événement, la stabilité du genre n'est plus un prérequis. Ahmed préfere Micheline «[a]vec les lunettes en homme, avec la perruque en femme» $[\mathrm{TD}, \mathrm{I} 8 \mathrm{I}])$. La tension n'est ainsi jamais là où elle devrait être. En quelque sorte, la crise telle que la vit le modèle bourgeois s'initie sur du vide - un vide "hystérisé». Si le couple, au commencement, est bien en difficulté, nul mensonge pour aller retrouver une quelconque maîtresse mais au contraire l'emprunt d'argent par l'un à l'autre pour aller se payer un tapin aux Tuileries. Si une mère tue bien son enfant, la question ne se pose pas de la légitimité d'un tel acte (sauf pour le père)... On pourrait multiplier les exemples qui révèlent que ce qui fait crise pour le boulevard ne fait jamais événement dans La Tour de la Défense. D'où le double mouvement du texte: l'un déceptif (ne pas répondre à l'horizon d'attente dominant), l'autre excessif (extrêmiser les possibles et produire de facto l'effet d'une «sensation d'effondrement léger, [un] sentiment d'irresponsabilité dont la cause serait, analogiquement du moins, une levée de censure $\left.{ }^{33} »\right)$.

Là où le boulevard, sous une forme ou une autre, joue de la responsabilité, comme moteur dramaturgique - il faut payer pour sa faute (adultère, concupiscence) -, Copi installe, dans une démarche très camp, l'irresponsabilité des figures en principe organisateur. Le processus d'enchaînement des faits n'est plus guidé et surdéterminé (principe de causalité) par l'idéologie dominante: il semble, en apparence, comme évidé de toute substance. Patrick Mauriès écrit que:

[...] le texte camp, entendu au sens large, est un texte fictif qui n'a de lui-même, à répondre de rien, telles les mécaniques de l'humoriste qui ne se mettent en action qu'auto-énergiquement; mais - et c'est là la source du malentendu - l'objet de ce texte est, la plupart du temps, un métalangage (existant ou stimulé), un code précis, une région du savoir qui se trouve soudain défonctionnalisée. Dévitalisée avec rigueur. ${ }^{34}$

33. P. Mauriès, Second Manifeste camp, op. cit., p. 89.

34. Ibid., p. I07. 
La citation décrit assez précisément le fonctionnement du texte de Copi : une mise en action auto-énergique, auto-instituante, enchâssée dans une hyponorme « [...] défonctionnalisée. Dévitalisée avec rigueur ». Dévitalisation littérale: ce qui arrive à la norme lorsqu'elle n'est plus incorporée par les sujets, lorsqu' elle n'est plus habitée par de la vie mais rendue à son arbitraire et à son historicité. Tout au plus peut-on reconnaître dans la pièce - et encore - un motif structurant: le désir de chacun-e, un désir forcément extravagant, exorbitant en tant qu'il est arraché à la servitude sociale, en tant que la représentation semble ignorer les principes et les présupposés de cette servitude. Une telle remarque prend toutefois le risque de dessiner un axe programmatique là où précisément Copi rompt avec toute contre-proposition (ce désir n'est pas un désir «libéré», socialisé différemment), toute possibilité de thématiser une perspective alternative. Le camp n'est "pas spirituel, et surtout n'est jamais vrai, n'est jamais épiphanie de vérité35", insiste Mauriès.

Le geste de Copi n'est donc pas tant d'importer une forme théâtrale (le boulevard) dans un registre culturel qui n'est pas le sien (la culture gay et lesbienne) mais de l'obliger à émigrer "hors de la sexualité dominante", une émigration sans autre horizon que le passage de frontières. À ce titre, la pièce n'est pas littéralement militante: elle ne préfigure aucune condamnation explicite, ne l'accompagne d'aucun discours valorisant et progressiste sur le "pédé» et la "gouine»: elle est de résistance, gangrenant la structure imitative dont peut se prévaloir le boulevard (dès le décor: celui-ci «est de l'ordre du décalque, sociologiquement intéressant, de la salle sur la scène $\left.{ }^{36} "\right)$ pour mettre en crise le caractère joué, fabriqué en toutes circonstances de l'ordre social et sexuel.

\section{Boiteuse et claudicante...}

La parodie est l'arme des fragiles, issue d'une vulnérabilité qui se sait fabriquée et saisie par le monde.

[...] ne pas lâcher son ennemi, le presser, le comprimer, le hanter, le doubler, être toujours à sa traîne, à ses basques, le tirer par la manche, le suivre comme son ombre. Car c'est ainsi [...] qu' on peut passer par-dessus l'ombre de l'adversaire, le précéder, le narguer; c'est surenchérir sur les actions, tenter de les forcer et de les dépasser pour lui arracher ce qu'il ne voulait pas donner, pour l'encercler, lui barrer, à son tour, toute issue et le contraindre à céder et à livrer ce qu'il retenait. ${ }^{37}$

Ce que retient le boulevard (et, par extension, le monde hétéropatriarcal) : l'arbitraire de ses présupposés. La démarche parodique, par écho, désigne

35. Ibid., p. I5.

36. M. Corvin, Le Théatre de boulevard, op. cit., p. II2. Il conclut néanmoins: «mais esthétiquement des plus pauvres".

37. F. Proust, De la résistance, Paris, Éditions du Cerf, 1997, p. 97. 
la norme: toute variation à partir de celle-ci, loin de la renforcer, peut en souligner le caractère fabriqué. La parodie serait ainsi subversive en ce qu'elle rapte voire usurpe le performatif «ceci est du boulevard» avec ce que celui-ci immanquablement induit (représentation instituante de codifications, normativisations) et qu'elle s'engage, pour paraphraser F. Collin, dans une praxis de la déconstruction où "le donné n'est pas considéré comme nul et non avenu, biffé d'un trait sous prétexte qu'il est "socialement construit", mais il est plutôt constamment raturé, déplacée ${ }^{38} »$. Cette rature constante fait obstacle, un temps du moins, à l'intuition naturalisante, dessaisissant chacun-e des grilles normatives d'intelligibilité du réel qu'elle se propose pourtant de copier.

Dès lors, cette résistance serait issue d'une "étrange logique [... ] celle d'un boitement et d'une claudication [...] Être sur tous les fronts sans chercher l'affrontement, batailler sans livrer bataille, guerroyer sans faire la guerre ${ }^{39}$ ", pour reprendre les mots de Françoise Proust dans son ouvrage De la résistance. On assiste ainsi à une dialecticité improbable: une négativité incarnée par une surenchère sur la positivité bourgeoise. À l'éclat lumineux de la négativité (ostensiblement exhibée, par exemple, par les situationnistes) répond ici la noirceur imprévue d'une positivité extrémisée découvrant le vide qu'elle mimait.

Tactiquement, Copi propose d'accompagner, de prolonger le jeu social là où le jeu en révélera l'artificialité et la contingence. Certes, à l'aune de l'efficacité dont serait comptable tout théâtre émancipateur, le théâtre de Copi ne pèse pas lourd, volontairement léger, comme un battement de faux cils ou un poignet cassé. Copi ne démontre pas, ne stigmatise pas l'oppression de l'hétéropatriarcat, il en désigne, réflexivement, les prétentions et les assignations.

Toutefois comme toute résistance, le danger est grand de l'anachronie. Judith Butler:

En soi, la parodie n'est pas subversive, il faut encore chercher à comprendre comment certaines répétitions parodiques sont vraiment perturbantes, sèment réellement le trouble, et lesquelles finissent par être domestiquées et circuler de nouveau comme des instruments de domination culturelle. Faire une typologie des actions ne serait vraisemblablement pas suffisant, car la déstabilisation parodique, dépend d'un contexte et de conditions de réception qui permettent d'entretenir les confusions subversives..$^{40}$

La résistance n'est ni abstraite ni intemporelle ni universelle. Elle s'ancre, tactique, dans un présent et un espace chaque fois singuliers. Ce qui, hier,

38. F. Collin, «Déconstruction ou destruction des genres», Contretemps: "Genre, classes, ethnies: identités, différences, égalités", $\mathrm{n}^{\circ} 7$, mai 2003, Éditions Textuel, p. 53.

39. F. Proust, De la résistance, op. cit., p. 182.

40. J. Butler, Trouble dans le genre, op. cit., p. 262. 
avait puissance subversive peut se révéler, aujourd'hui, allié de l'oppression. Telle n'est pas la moindre limite de ce théâtre, de cette écriture: être exhibé comme une provocation productrice qu'il n'est déjà plus, déjà avalé, déjà digéré. Il en va de même pour le rire désormais fétichisé comme arme contre les puissants, là où si souvent il redouble et conforte leur tyrannie. En I977, Guy Hocquenghem concluait un article sur Copi par une hypothèse:

Curieusement, ce que décrit Copi est déjà archéologique. [...] Peut-être que la sensibilité folle [...] cet ars erotica féroce et subtil aussi fragile qu'une fleur en papier japonaise et aussi acéré qu'un certain cri aigu, c'est déjà de l'art, ce n'est plus, ou si peu, de la vie. ${ }^{4 \mathrm{I}}$

À cette aune, la création de pensées politiques homogènes aux situations plus ou moins neuves qui se présentent permet d'acérer et d'armer nos lectures: refuser le basculement dans la sphère esthétique de ce qui tendait à s'en extraire pour y déceler (ou non) hic et nunc des potentialités politiques radicales. Une lecture en quelque sorte "léniniste» des œuvres: reliant leur tactique à leur conjoncture. Ainsi confrontées, et toutes archéologiques qu'elles aient pu paraître, toutes œuvres qu'elles soient devenues, les pièces de Copi n'en sont pas moins encore, sous certaines conditions, des armes efficaces pour troubler l'Ordre. Des pièces de résistances donc mais, et là réside peut-être sa difficulté principale, dans un monde où «la position hégémonique est celle de la résistance», où le discours même de la "résistance» est progressivement devenu « la norme et $[. .$.$] le principal obstacle à la naissance d'un discours qui remettrait$ véritablement en cause les conditions dominantes ${ }^{42} »$.

4I. G. Hocquenghem, «Invitation au délire», La Dérive homosexuelle, Jean-Pierre Delarge, I977, p. I44.

42. S. Zizek, La Marionnette et le nain. Le christianisme entre perversion et subversion, Paris, Seuil, «La couleur des idées», 2006, p. 196. 